\title{
Active vs. reactive threat responding is associated with differential c-Fos expression in specific regions of amygdala and prefrontal cortex
}

\author{
Raquel C.R. Martinez, ${ }^{1,2}$ Nikita Gupta, ${ }^{2}$ Gabriel Lázaro-Muñoz, ${ }^{2}$ Robert M. Sears, ${ }^{2}$ \\ Soojeong Kim, ${ }^{3}$ Justin M. Moscarello, ${ }^{2}$ Joseph E. LeDoux, ${ }^{2,3}$ and Christopher K. Cain ${ }^{2,3,4}$ \\ ${ }^{1}$ University of Sao Paulo, Medical School, Surgery Department (LIM 26 HCFMUSP), Sao Paulo 01246-903, Brazil; ${ }^{2}$ Center for Neural \\ Science, New York University, New York 10003, USA; ${ }^{3}$ Emotional Brain Institute, Nathan Kline Institute for Psychiatric Research, \\ Orangeburg, New York 10962, USA
}

\begin{abstract}
Active avoidance (AA) is an important paradigm for studying mechanisms of aversive instrumental learning, pathological anxiety, and active coping. Unfortunately, AA neurocircuits are poorly understood, partly because behavior is highly variable and reflects a competition between Pavlovian reactions and instrumental actions. Here we exploited the behavioral differences between good and poor avoiders to elucidate the AA neurocircuit. Rats received Sidman AA training and expression of the activity-dependent immediate-early gene $c$-fos was measured after a shock-free AA test. Six brain regions with known or putative roles in AA were evaluated: amygdala, periaqueductal gray, nucleus accumbens, dorsal striatum, prefrontal cortex (PFC), and hippocampus. Good avoiders showed little Pavlovian freezing and high AA rates at test, the opposite of poor avoiders. Although c-Fos activation was observed throughout the brain, differential activation was found only in subregions of amygdala and PFC. Interestingly, c-Fos correlated with avoidance and freezing in only five of 20 distinct areas evaluated: lateral amygdala, central amygdala, medial amygdala, basal amygdala, and infralimbic PFC. Thus, activity in specific amygdala-PFC circuits likely mediates the competition between instrumental actions and Pavlovian reactions after AA training. Individual differences in AA behavior, long considered a nuisance by researchers, may be the key to elucidating the AA neurocircuit and understanding pathological response profiles.
\end{abstract}

Many anxiety disorders are characterized by abnormal fear and avoidance following aversive experience. This usually means excessive fear, failure to suppress fear, or adoption of avoidance patterns that interfere with normal activities. These symptoms are thought to arise from maladaptive threat processing in brain circuits tasked with defense and coping (LeDoux 2012).

Threat processing has been intensely studied with animal models of Pavlovian learning, like fear (threat) conditioning and extinction. However, much less is known about the neural circuits mediating active avoidance (AA), a form of instrumental conditioning that depends on Pavlovian conditioning (Mowrer and Lamoreaux 1946; Rescorla and Solomon 1967; Levis 1989; McAllister and McAllister 1991). In AA, subjects learn that specific actions will escape threats (conditioned stimuli [CSs]) and prevent harm (unconditioned stimuli [USs]). AA mechanisms evolved because they are, on balance, adaptive. Unlike Pavlovian reactions, which prepare animals for impending harm, proactive AA responses allow subjects to control exposure to threats and avert pain or injury altogether. AA responding also blocks the expression of Pavlovian reactions (Solomon and Wynne 1954; Baum and Poser 1971; Choi et al. 2010; Lazaro-Munoz et al. 2010), which, in humans, are associated with unpleasant feelings like fear (Delgado et al. 2006). And unlike passive avoidance, AA can be an effective way to remain safe without disengaging from environments that include danger (Cain and LeDoux 2008). Though avoidance can become pathological when it interferes with healthy activity, a different and profound form of pathology can

\section{${ }^{4}$ Corresponding author}

E-mail ccain@nki.rfmh.org

Article is online at http://www.learnmem.org/cgi/doi/10.1101//m.031047.113. result when threat reactions, like freezing, prevent the expression of adaptive avoidance responses.

Most animals successfully acquire AA; however, some fail to show AA responses even after extensive training. Although "poor avoiders" are usually excluded from analyses of AA under the assumption that they fail to learn (e.g., Bolles and Popp 1964), we recently demonstrated that poor avoidance reflects a performance deficit, not a learning deficit. Indeed, poor avoiders freeze excessively, and lesions of the central amygdala that eliminate Pavlovian freezing rescue AA responding without further training (Choi et al. 2010; Lazaro-Munoz et al. 2010). In the present study, rather than excluding poor avoiders, we exploited these stark behavioral differences to help elucidate the AA neurocircuit. We reasoned that good and poor avoidance result from differential recruitment of brain circuits associated with instrumental actions and Pavlovian reactions. By contrasting the neural activity observed in these groups we were able to examine neurocircuits associated with AA expression while circumventing tricky instrumental control issues that have hampered previous research (Church 1989; Nikolaev et al. 1992). Critically, this approach aligns with recent efforts to focus animal models of anxiety on atypical, rather than typical, responding (Yehuda et al. 2006; Yehuda and LeDoux 2007; Sotres-Bayon et al. 2008). Since only some individuals develop pathological anxiety, approaches that focus on individual differences are more likely to provide key insights into neural processes awry in human disorders.

Forty-two rats received seven Sidman AA training sessions and good $(n=5)$ and poor $(n=6)$ avoiders were selected for comparison to each other and to nontrained box controls $(n=5)$. Rats were sacrificed 90 min after a shock-free AA test. c-Fos was measured as an index of neuronal activity, because expression of 
this immediate-early gene (IEG) product has proven useful in past studies of behavioral neurocircuits (e.g., Knapska et al. 2007). c-Fos was quantified in six brain regions with known or putative roles in AA: amygdala, medial prefrontal cortex (mPFC), periacqueductal gray (PAG), dorsal striatum, nucleus accumbens (NAC), and hippocampus. This approach identified only five discrete subregions of amygdala and mPFC that were associated with good vs. poor avoidance, suggesting that these regions represent critical components of the AA neurocircuit that contribute to response variability.

\section{Results}

\section{Behavior}

AA training produced both good avoiders and poor avoiders (Fig. 1); good avoiders dramatically increased shuttling by session 7 and poor avoiders did not (Group $\times$ Session, $F_{(6,54)}=10, P<$ 0.01). During the shock-free test, poor avoiders froze more $\left(t_{(9)}=3.9, P<0.01\right)$ and shuttled less $\left(t_{(9)}=4.1, P<0.01\right)$ than good avoiders. Freezing and shuttling correlated negatively during the test $\left(r^{2}=0.67, F_{(1,9)}=18, P<0.01\right)$.

\section{c-Fos expression}

c-Fos density after AA testing was compared for box controls, good avoiders, and poor avoiders in amygdala, medial PFC, NAC, dorsal striatum, PAG, and hippocampus. Key subregions, as delineated by Pitkänen et al. (1997), were analyzed separately. Differences along the AP axis (relative to bregma) are reported only if the three-way (Group $\times$ Subregion $\times$ AP-level) interactions were statistically significant. Significant differential c-Fos expression effects are detailed in Figure 2, and all results are summarized in Figure 3.

Six amygdala nuclei were assessed together: lateral (dorsal and ventral subregions [LA $A_{D}$ and $L A_{V}$, respectively]), basal (anterior, posterior, and ventral subregions $\left[\mathrm{BA}_{\mathrm{A}}, \mathrm{BA}_{\mathrm{P}}\right.$, and $\mathrm{B} \mathrm{A}_{\mathrm{V}}$, respectively]), accessory basal (AB), medial (dorsal and ventral subregions $\left[\mathrm{ME}_{\mathrm{D}}\right.$ and $\mathrm{ME}_{\mathrm{V}}$, respectively]), central (lateral and medial subregions $\left[\mathrm{CE}_{\mathrm{L}}\right.$ and $\mathrm{CE}_{\mathrm{M}}$, respectively]) and cortical $(\mathrm{CO})$. The three-way interaction between Group, Subregion, and AP-level was not significant $\left(F_{(46,299)}=1.2, P=0.2\right)$. Large c-Fos differences were observed throughout the amygdala; effects of Group $\left(F_{(2,13)}=318\right)$, Subregion $\left(F_{(10,130)}=88\right)$, and their interaction $\left(F_{(20,130)}=2.1, P<0.01\right)$ were all significant. c-Fos density was higher in all amygdala subregions for AA-trained rats (good and poor avoiders) compared to box controls ( $P$ values $<0.05$ ).

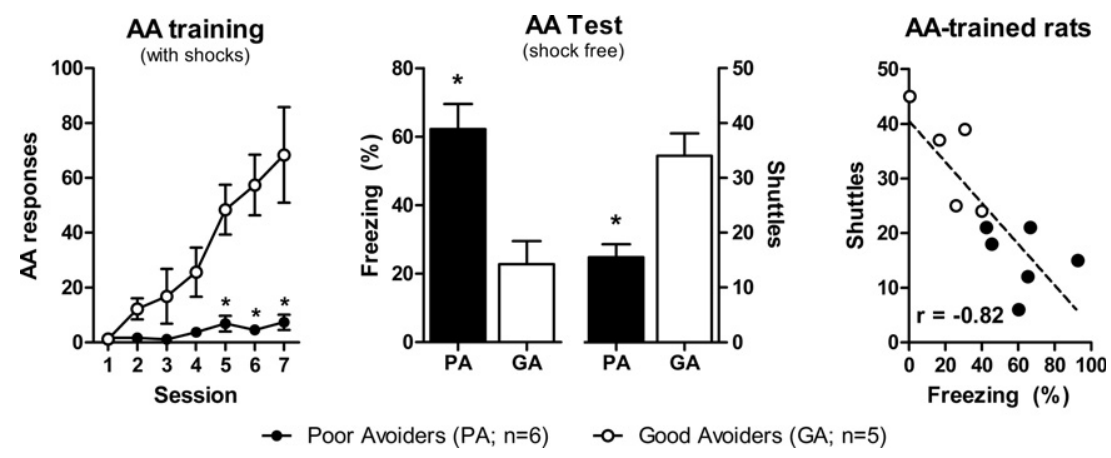

Figure 1. Inverse relationship between Pavlovian and instrumental responses in Good vs. Poor avoiders. (Left) Mean AA responding during seven daily training sessions. (Middle) Mean freezing and shuttling during the final shock-free test session. (Right) Negative correlation between freezing and shuttling for individuals during the test session. (AA) Active avoidance, (GA) good avoiders, (PA) poor avoiders. $(*) P<0.05$ vs. GA group. Error bars = SEM.
With the exception of $\mathrm{AB}\left(t_{(130)}=1.8, P=0.07\right)$ and $\mathrm{CO}\left(t_{(130)}=\right.$ $0.6, P=0.56)$, c-Fos differed between good and poor avoiders in every amygdala subregion $\left(t_{(130)}\right.$ values $>2.4, P$ values $\left.<0.05\right)$. c-Fos correlated negatively with freezing during the final test in five amygdala subregions: $\mathrm{LA}_{\mathrm{D}}\left(-3.8 \mathrm{~mm}\right.$ from bregma, $r^{2}=$ 0.76, $\left.F_{(1,9)}=28.7, P<0.01\right), \mathrm{ME}_{\mathrm{D}}\left(-3.3 \mathrm{~mm}\right.$ from bregma, $r^{2}=$ $\left.0.37, F_{(1,9)}=5.2, P<0.05\right), \mathrm{ME}_{\mathrm{V}}\left(-2.3 \mathrm{~mm}\right.$ from bregma, $r^{2}=$ $\left.0.52, F_{(1,9)}=9.9, P=0.01\right), \mathrm{BA}_{\mathrm{P}}\left(-3.3 \mathrm{~mm}\right.$ from bregma, $r^{2}=$ $\left.0.58, F_{(1,9)}=12.6, P<0.01\right)$, and $\mathrm{CE}_{\mathrm{L}}(-2.8 \mathrm{~mm}$ from bregma, $\left.r^{2}=0.52, F_{(1,9)}=9.6, P<0.05\right)$. Conversely, c-Fos correlated positively with shuttling in seven amygdala subregions: $\mathrm{LA}_{\mathrm{D}}(-3.8$ $\mathrm{mm}$ from bregma, $\left.r^{2}=0.62, F_{(1,9)}=14.5, P<0.01\right), \mathrm{LA}_{\mathrm{V}}(-3.3$ $\mathrm{mm}$ from bregma, $\left.r^{2}=0.42, F_{(1,9)}=6.5, P<0.05\right), \mathrm{ME}_{\mathrm{V}}(-2.3$ $\mathrm{mm}$ from bregma, $\left.r^{2}=0.53, F_{(1,9)}=10.3, P=0.01\right), \mathrm{BA}_{\mathrm{P}}(-3.3$ $\mathrm{mm}$ from bregma, $\left.r^{2}=0.38, F_{(1,9)}=5.4, P<0.05\right), \mathrm{AB}(-3.3$ $\mathrm{mm}$ from bregma, $\left.r^{2}=0.46, F_{(1,9)}=7.6, P<0.05\right), \mathrm{CE}_{\mathrm{L}}(-2.8$ $\mathrm{mm}$ from bregma, $\left.r^{2}=0.75, F_{(1,9)}=27.4, P<0.01\right)$, and $\mathrm{CE}_{\mathrm{M}}$ $\left(-2.8 \mathrm{~mm}\right.$ from bregma, $\left.r^{2}=0.44, F_{(1,9)}=7.1, P<0.05\right)$. c-Fos never correlated positively with freezing or negatively with shuttling. Thus, only four amygdala subregions $\left(\mathrm{LA}_{\mathrm{D}}, \mathrm{CE}_{\mathrm{L}}, \mathrm{ME}_{\mathrm{V}}\right.$, and $\mathrm{BA}_{\mathrm{P}}$ ) showed differential c-Fos expression (good vs. poor avoiders) that correlated with both behavioral measures (freezing and shuttling) during the final test (Fig. 2).

Two medial PFC subregions were assessed: prelimbic (PL) and infralimbic (IL). Large c-Fos differences were observed throughout $\operatorname{PFC}\left(F_{(2,13)}=108, P<0.01\right)$ and AA-trained rats showed higher c-Fos expression than box controls in both subregions ( $P$ values $<0.01)$. The three-way interaction between Group, Subregion, and AP-level was statistically significant $\left(F_{(4,26)}=3.6, P=0.02\right)$. Post-hoc contrasts revealed c-Fos differences between good and poor avoiders in both $\mathrm{mPFC}$ subregions at $+3.72 \mathrm{~mm}$ from bregma (PL, $\left.t_{(24)}=3.4, P<0.01 ; \mathrm{IL}, t_{(24)}=3.7, P<0.01\right)$ but smaller differences at $+3.0 \mathrm{~mm}\left(\mathrm{PL}, t_{(24)}=2.1, P=0.04\right)$, especially in IL $\left(t_{(24)}=1.4, P=0.2\right)$. c-Fos correlated negatively with freezing during the final test in IL only $\left(+3.72 \mathrm{~mm}\right.$ from bregma, $r^{2}=$ $\left.0.40, F_{(1,9)}=6.0, P<0.05\right)$. Conversely, c-Fos density correlated positively with shuttling in PL $\left(+3.72 \mathrm{~mm}\right.$ from bregma, $r^{2}=$ 0.51, $\left.F_{(1,9)}=9.4, P<0.01\right)$ and IL $\left(+3.72 \mathrm{~mm}\right.$ from bregma, $r^{2}=$ $\left.0.70, F_{(1,9)}=20.6, P<0.01\right)$. c-Fos never correlated positively with freezing or negatively with shuttling. Thus, only anterior IL showed differential c-Fos expression that correlated with both behavioral measures during the final test (Fig. 2).

Subregions of four additional brain areas were also assessed: PAG (dorsal and ventral $\left[\mathrm{PAG}_{\mathrm{D}}\right.$ and $\mathrm{PAG}_{\mathrm{V}}$, respectively]), NAC (core and shell $\left[\mathrm{NAC}_{\mathrm{C}}\right.$ and $\mathrm{NAC}_{\mathrm{S}}$, respectively]), dorsal striatum (dorsomedial and dorsolateral [DMS and DLS, respectively]), and hippocampus (CA1). Group differences in c-Fos density were found in PAG $\left(F_{(2,13)}=16.5, P<0.01\right)$, NAC $\left(F_{(2,13)}=\right.$ $10.3, P<0.01)$, and dorsal striatum $\left(F_{(2,13)}=43.0, P<0.01\right)$, but not hippocampus $\left(F_{(2,13)}=0.21\right)$. However, in each case these were driven by large differences between AA-trained (good and poor avoiders) and nontrained (box control) rats $\left(t_{(13)}>2.9, P\right.$ values $\left.\leq 0.01\right)$. Further, group differences did not vary according to subregion or AP level $(P$ values for Group $\times$ Subregion and Group $\times$ Subregion $\times$ AP-level interactions all $>0.05$ ) and post-hoc contrasts revealed no c-Fos differences between good and poor avoiders $\left(t_{(13)}\right.$ values $<1.9, P$ values $>0.08$ ). DLS was the only region to show a trend toward differential c-Fos expression for good vs. poor avoiders $\left(t_{(13)}=\right.$ 

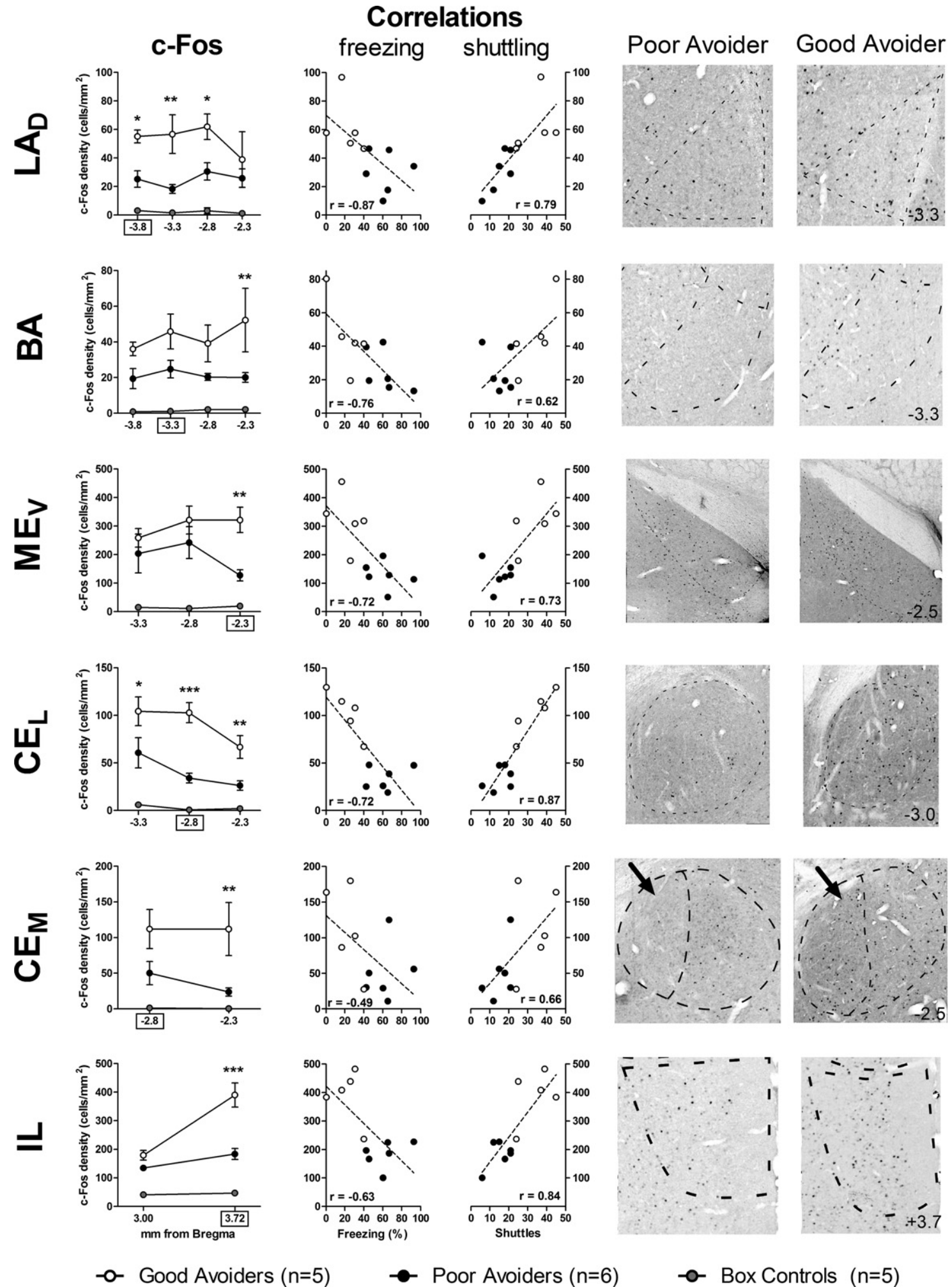

Poor Avoiders ( $n=6)$

Box Controls ( $n=5)$

Figure 2. Post-test c-Fos expression is associated with Good vs. Poor avoidance in amygdala and prefrontal cortex. (Left) Mean density of c-Fos ${ }^{+}$cells for Good avoiders, Poor avoiders and box controls at multiple anterior-posterior (AP) levels (relative to bregma). (Middle) In each region shown, c-Fos correlated negatively with freezing and positively with shuttling. With the exception of freezing and $\mathrm{CE}_{\mathrm{M}} \mathrm{C}$-Fos, all correlations are statistically significant. (Right) Representative photomicrographs depicting c-Fos in Good and Poor avoiders (approximate AP level, in mm from bregma, shown in bottom right of Good avoider images). (LA $A_{D}$ ) Dorsal division of lateral amygdala, $(B A)$ basal amygdala, $\left(C E_{L}\right)$ lateral division of central amygdala, (CE ${ }_{M}$ ) medial division of central amygdala, (MEV) ventral division of medial amygdala, (IL) infralimbic prefrontal cortex. $\left.{ }^{*}\right) P<0.05,\left({ }^{* *}\right) P<0.01,\left({ }^{* * *}\right) P<$ 0.001 for Good vs. Poor avoider contrasts at specific AP levels. AP level for correlations indicated by $x$-axis boxes on c-Fos graphs. Error bars $=S E M$. 


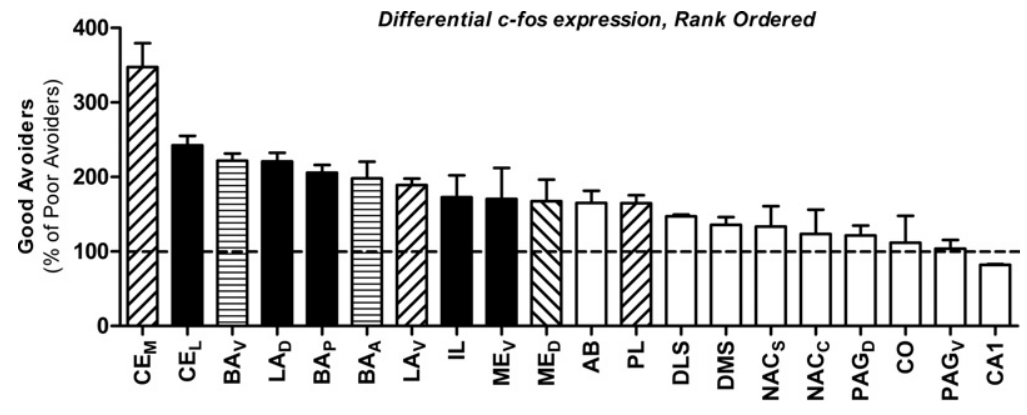

KEY

= FOS differs between groups and correlates with both behaviors
$=$ FOS differs between groups and correlates with freezing
$=$ FOS differs between groups and correlates with shuttling
$=$ FOS differs between groups but correlates with neither behavior
$=$ FOS doesn't differ between groups

Figure 3. Summary of differential c-Fos expression effects. Good avoider values were normalized using Poor avoider group means and effects are expressed as a percentage of Poor avoiders (mean \pm SEM). Differential c-Fos expression in all 20 brain regions examined, rank ordered by effect magnitude. Bar shading indicates statistical significance of c-Fos expression differences (Good vs. Poor avoiders), and correlations between c-Fos expression and behavior (see Key). The absence of differential expression is indicated by the dashed line at $100 \%$. (CE) Central amygdala, (BA) basal amygdala, (LA) lateral amygdala, (ME) medial amygdala, (AB) accessory basal amygdala, (CO) cortical amygdala, (IL) infralimbic prefrontal cortex, (PL) prelimbic prefrontal cortex, (PAG) periacqueductal gray, (DMS) dorsomedial striatum, (DLS) dorsolateral striatum, (NAC) nucleus accumbens, (CA1) hippocampal CA1 area, (A) anterior, $(P)$ posterior, $(D)$ dorsal, $(V)$ ventral, $(M)$ medial, $(L)$ lateral, $(C)$ core, $(S)$ shell. Error bars = SEM.

$1.8, P=0.09)$. Further, c-Fos did not correlate with either behavioral measure in any of these regions or subregions $\left(F_{(1,9)}\right.$ values $<1.9, P$ values $>0.19$ ).

\section{Discussion}

Active avoidance (AA) is an important instrumental learning paradigm with clear relevance to the development and treatment of human pathological anxiety. In AA, subjects learn to suppress Pavlovian reactions and emit actions that limit exposure to threatening or harmful stimuli. However, in contrast to other conditioning paradigms like fear conditioning or passive avoidance, very little is known about the neurobiological mechanisms mediating AA-most likely because a defined neurocircuit is lacking. There are many reasons for this, but a major reason may be that AA training induces both Pavlovian reactions, like freezing, and instrumental actions, like shuttling, that are incompatible, competing behaviors (Lazaro-Munoz et al. 2010). Thus, AA responding is not a pure readout of AA learning, which can confuse efforts to identify an underlying neurocircuit.

Instead of excluding poor avoiders from analysis, we exploited the stark behavioral differences between good and poor avoiders to elucidate the AA neurocircuit. Good avoiders displayed high AA rates and low freezing following a moderate amount of training, whereas poor avoiders showed an opposite pattern. Of the 20 brain regions examined, only five showed differential c-Fos expression that correlated with both behaviors. Four were subregions of amygdalar nuclei-dorsal LA, ventral $\mathrm{ME}$, lateral $\mathrm{CE}$, and posterior BA. The fifth was a subregion of PFC, anterior IL. In each of these regions, c-Fos correlated positively with shuttling and negatively with freezing. Although c-Fos induction certainly occurred in many other regions examined, it was not related to the behavioral differences observed between good and poor avoiders following identical training and testing. These results support the notion that activity in specific amygdala-PFC neurocircuits mediates the suppression of Pavlovian reactions and the transition to instrumental actions with AA training.
Many studies have examined IEG activation related to aversive conditioning; however, the vast majority used Pavlovian fear conditioning or passive avoidance, and have produced inconsistent results (for discussions, see Scicli et al. 2004; Knapska et al. 2007). We are aware of no studies examining IEG activation with Sidman AA, but several have examined signaled AA (Nikolaev et al. 1992; Duncan et al. 1996; Savonenko et al. 1999; Radwanska et al. 2002; Saha and Datta 2005). Unfortunately, the results fail to paint a clear picture of the AA neurocircuit. The most consistent findings are of elevated activity in amygdalar LA, BA, and ME nuclei following AA training. One study also found elevated c-Fos in $\mathrm{mPFC}$ following AA training (Duncan et al. 1996). However, most of these studies identified additional brain regions of presumably equal importance, such as the cingulate cortex, lateral septum, and various hypothalamic nuclei (Duncan et al. 1996; Saha and Datta 2005), with little overlap between studies.

At first glance, several findings appear to be at odds with our results. For example, some studies identified CO and PAG as important for AA, and none found significant activation in CE. However, several differences between our study and prior studies may account for these apparent inconsistencies. First, we examined activation patterns following a moderate amount of AA training (seven sessions). All but one of the above studies used a single training session. Thus, our protocol clearly identified good and poor avoiders before the analysis, whereas the others could not take advantage of this distinction. This also makes it likely that our analysis primarily implicates performance mechanisms, whereas the above studies may be more relevant to learning. Second, all of the above studies used control conditions (e.g., naive or box control) that provide a poor contrast with AA training. Selecting an appropriate associative control is, indeed, difficult for AA studies (for discussions, see Church 1989; Nikolaev et al. 1992); however, comparisons with untrained controls lead to identification of any region activated by training, regardless of any specific role in AA learning or performance. This problem may have been magnified by the use of test sessions that included shock presentations. Our design allowed us to circumvent these difficulties by contrasting good and poor avoiders following identical training and testing. It should be noted that we also found significant c-Fos activation in many of the same regions identified in past studies (by comparison to box controls), sometimes massive activation (e.g., cortical amygdala). However, in the absence of evidence for differential activity between good and poor avoiders, it is difficult to conclude that these regions contribute to AA performance. Last, previous studies failed to evaluate potentially important differences within subregions of brain nuclei (e.g., Nikolaev et al. 1992).

It is interesting, and somewhat unexpected, that c-Fos correlated positively with shuttling and negatively with freezing in all of the regions depicted in Figure 2. We expected to find some regions where excessive freezing in poor avoiders was associated with higher c-Fos expression, perhaps regions mediating Pavlovian freezing, like medial CE or ventral PAG (Hitchcock and Davis 1991; Kim et al. 1993). The positive relationship between c-Fos 
and avoidance in medial CE is a bit puzzling, especially since CE is not necessary for avoidance and CE lesions facilitate avoidance when freezing interferes (Choi et al. 2010; Lazaro-Munoz et al. 2010). One possibility is that heightened activity related to freezing is more likely to be observed very early in training, when Pavlovian reactions are rapidly acquired and stored. Another possibility is that different cell populations within these brain regions contribute to AA or freezing (e.g., excitatory vs. inhibitory neurons), even if the region is not essential for AA (e.g., CE). Finally, Sidman AA is a difficult, multi-faceted task. Performance depends on Pavlovian learning, instrumental learning, interval timing, safety signals, and specific action sequences. Successful negotiation of this task depends on the coordinated recruitment of circuit components mediating these diverse functions. Thus, poor avoiders may show less overall c-Fos activity because they fail to recruit these interdependent processes and fail to experience avoidance feedback cues.

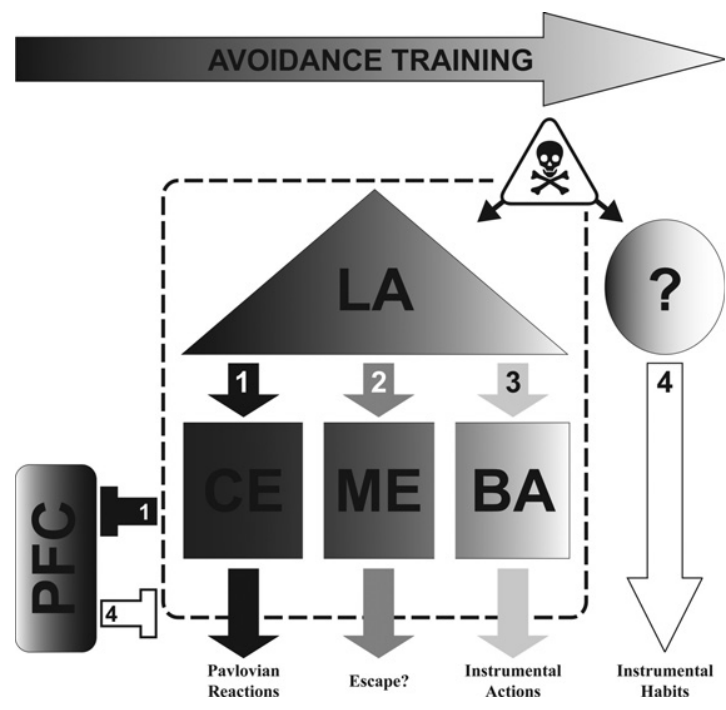

Figure 4. Working model: neural circuit mediating the progression from Pavlovian reactions to instrumental actions with AA training. Dark shading represents primarily Pavlovian processes early in training. Lighter shading represents the emergence of instrumental processes with continued training. (1) Early in training, dorsal LA learns and stores Pavlovian CS-US associations. Subsequent threats (CSs) activate LA and $\mathrm{CE}$, triggering activity in downstream regions mediating Pavlovian reactions (e.g., freezing). As training progresses, (2) subjects first learn to efficiently escape the US and then (3) to escape the CS and prevent the US (avoidance). We hypothesize that ME is important for initial escape learning that prepares the subject to avoid. Once the "escape" response occurs during the $\mathrm{CS}$, acquisition of the avoidance contingency can proceed and strengthen, via connections from LA to BA. After a moderate amount of training, threats activate LA and trigger both Pavlovian and instrumental memories that compete for control of behavior, via CE and BA outputs, respectively. PFC may also play an important role in the balance between Pavlovian reactions and instrumental actions. (1) Early in training, PFC is likely suppressed by heightened amygdala activity. However, akin to extinction, PFC may suppress Pavlovian threat reactions later in training to allow for the acquisition and expression of avoidance. (4) Ultimately, with overtraining the entire amygdala becomes unnecessary and AA expression depends on extra-amygdala regions (not yet identified). Although parts remain speculative, available lesion data and present c-Fos results are consistent with this model. Poor avoidance could result from excessive $L A \rightarrow C E$ activation of Pavlovian reactions, failure of PFC to suppress CE outputs, or failure to transfer AA control to extra-amygdala regions with overtraining. (LA) Lateral amygdala, (BA) basal amygdala, (CE) central amygdala, (ME) medial amygdala, (PFC) prefrontal cortex, (CS) conditioned stimulus, (US) unconditioned stimulus, (AA) active avoidance.
Our findings may help refine models of the AA neurocircuitry (see Fig. 4). For instance, LA, BA, and CE have all been implicated in Pavlovian freezing, instrumental AA, or their competition (Hitchcock and Davis 1991; Poremba and Gabriel 1999; AngladaFigueroa and Quirk 2005; Choi et al. 2010; Lazaro-Munoz et al. 2010). However, our c-Fos results highlight the potential importance of specific subregions within these nuclei. For instance, $\mathrm{LA}_{\mathrm{D}}$, rather than the whole basolateral complex, may be particularly important for AA, as it is for Pavlovian conditioning (Repa et al. 2001; Han et al. 2007). Interestingly, our findings indicate an important role for $\mathrm{CE}_{\mathrm{L}}$ in $\mathrm{AA}$, even though $\mathrm{CE}_{\mathrm{M}}$ is more closely associated with threat responding (Hitchcock and Davis 1991). Several recent papers suggest that a population of inhibitory neurons in $\mathrm{CE}_{\mathrm{L}}$ may be critical for suppressing threat reactions like freezing (Ciocchi et al. 2010; Gozzi et al. 2010; Haubensak et al. 2010). We hypothesize that differential c-Fos expression in $\mathrm{CE}_{\mathrm{L}}$ occurs in these GABAergic " $\mathrm{CS}_{\text {off }}$ " cells with AA. Finally, posterior regions of BA may also play a special role. Thus, our findings may help identify specific cell populations in amygdala that contribute to response variability following AA training.

Our analyses also highlight two new regions that may be important for AA: anterior IL-PFC and ventral ME. IL-PFC has a wellestablished role in fear extinction, where nonreinforced CS exposures lead to inhibitory learning and suppression of Pavlovian reactions. The involvement of IL-PFC in AA seems consistent with fear extinction findings (Herry and Mons 2004; Hefner et al. 2008; Sotres-Bayon et al. 2008); perhaps IL-PFC plasticity is necessary to suppress amygdala-mediated reactions like freezing and allow for AA learning and expression. Very recent findings support this hypothesis; damage or protein synthesis blockade in IL-PFC both impair signaled AA (Moscarello and LeDoux 2013). It should be noted that differential activity in IL-PFC could also reflect differences in extinction of Pavlovian vs. instrumental responding in poor vs. good avoiders, since our AA test session was shock-free. Last, a large difference in c-Fos expression between good and poor avoiders was found in $\mathrm{ME}_{\mathrm{V}}$. ME has no known role in AA behavior; however, it has never been directly studied. Interestingly, ME activity is associated with escape behavior (e.g., Herdade et al. 2006), and learned escape (of the US and CS) may be a critical step in the transition from Pavlovian reactions to instrumental actions with AA training (Bolles 1969).

In summary, we evaluated Pavlovian freezing, instrumental shuttling, and brain c-Fos expression in good vs. poor avoiders. This approach identified only five discrete subregions of amygdala and PFC where neural activity correlated with both reactive (Pavlovian) and proactive (instrumental) defensive behavior. These findings provide converging evidence, from animals with intact brains, that response competition following AA training depends critically on specific mPFC-amygdala circuits. Naturally occurring variations in amygdala-mPFC activity with AA resemble those found in good vs. poor extinguishers (e.g., Hefner et al. 2008). Thus, AA and extinction may share a common mechanism that contributes to a "poor-coping" phenotype when dysfunctional.

\section{Materials and Methods}

\section{Subjects/apparatus}

Male Sprague-Dawley rats (Hilltop Labs, Scottdale, PA) weighing 250-300 g were maintained on a 12:12-h light cycle with ad libitum food and water. Experiments were approved by the NYUIACUC and conformed to NIH Guidelines. Behavioral training/ testing was conducted in two-way shuttleboxes controlled by GraphicState software (Coulbourn Instruments). Shuttling was registered by infrared beams and final tests were recorded to 
DVD for freezing analyses. Freezing was assessed during the first 2 min of shock-free tests by observers blind to group specification.

\section{Sidman AA protocol}

Sidman AA was chosen over signaled AA because it is a more difficult protocol that produces a higher percentage of poor avoiders (Choi et al. 2010; Lazaro-Munoz et al. 2010). Forty-two rats received seven daily 25 -min training sessions (excluding weekends). Five good avoiders and six poor avoiders were selected for further analysis, as described previously (Lazaro-Munoz et al. 2010). Briefly, shuttling between compartments delayed the delivery of scrambled footshock USs ( $1 \mathrm{~mA}, 0.5 \mathrm{sec})$ by $30 \mathrm{sec}$ (R-S interval). In the absence of shuttling, US delivery occurred every 5 sec (S-S interval). R-S interval shuttles were considered avoidance responses and S-S interval shuttles were escape responses. All shuttles produced 0.3-sec feedback stimuli (houselight blink). Twenty-four hours after session 7, good and poor avoiders were given a shockfree test, otherwise identical to training. Five additional boxcontrol rats received equivalent exposure to the chambers, but no shocks.

\section{Perfusion}

Ninety minutes after the AA test, rats were anesthetized with chloral hydrate $(150 \mathrm{mg} / \mathrm{kg})$ and perfused transcardially with $4 \%$ paraformaldehyde in $0.1 \mathrm{M}$ phosphate buffer $(\mathrm{pH} 7.4)$. Brains were removed, bathed in paraformaldehyde for $3 \mathrm{hr}$, and then transferred to a $30 \%$ sucrose $/ 0.1 \mathrm{M}$ phosphate buffer at $4^{\circ} \mathrm{C}$ overnight. Frozen sections $(40 \mu \mathrm{m})$ were then cut with a sliding microtome along the frontal plane.

\section{c-Fos immunoreactivity}

Brain sections were processed with anti-c-Fos antiserum raised in rabbit (Ab-5, Calbiochem, lot-D07099, dilution =1:20,000). Primary antiserum was localized using an avidin-biotin complex system (ABC; Vector Laboratories). Briefly, sections were incubated in biotinylated goat anti-rabbit IgG solution (Vector Laboratories), then placed in mixed avidin-biotin horseradish peroxidase complex solution (ABC Elite Kit; Vector Laboratories) (90 min/ step at $22^{\circ} \mathrm{C}$ ). The peroxidase complex was incubated in chromogen solution containing $0.02 \% 3,3^{\prime}$-diaminobenzidine tetrahydrochloride (DAB) (Sigma) with 0.3\% nickel-ammonium sulfate in $0.05 \mathrm{M}$ Tris-buffer ( $\mathrm{pH} 7.6)$, then in chromogen solution with hydrogen peroxide (1:3000) (10 $\mathrm{min} / \mathrm{step})$. The DAB reaction was halted by extensive washing in PBS ( $\mathrm{pH}$ 7.4). Sections were mounted on gelatin-coated slides, dehydrated, and coverslipped with DPX (Sigma). An adjacent reference series was Nissl stained.

\section{Quantification}

c-Fos-immunoreactive neurons were evaluated from $10 \times$ images (Nikon Eclipse 80i-microscope with Digital Camera DXM1200F). Regions of interest (ROIs) were manually delineated, guided by adjoining Nissl-stained sections and a rat brain atlas (Paxinos and Watson 2005). Border delineation, cell counting, and area measurements were done with Image-J software (Version 1.44b). At least two measurements along the anterior-posterior (AP) axis were made for each ROI. ROIs were chosen based on hypothesized or known roles in Pavlovian fear, AA, or appetitive instrumental conditioning (Cain and LeDoux 2008).

\section{Statistical analyses}

Avoidance training data were analyzed using two-way (Group $\times$ Session) repeated measures ANOVAs with Bonferroni post-tests. Shock-free tests were analyzed with two-tailed unpaired $t$-tests. Since avoidance differences during the Session 8 test occurred mainly during the first $10 \mathrm{~min}$ of testing, all analyses reflect behavior during this period only. Box controls were included as a control for c-Fos analyses, but not behavioral analyses, because they were never shocked, never froze, and, by definition, could not emit AA responses. Group effects on c-Fos density in major brain regions (amygdala, mPFC, PAG, NAC, dorsal striatum, and hippocampus) were evaluated with separate mixed model ANOVAs (SAS PROC MIXED procedure with ESTIMATE statements for interaction contrasts between groups). Since major brain regions were usually divided into subregions, and multiple measures were taken along the AP axis, AP levels were nested in subregions, and subregions were nested in subjects (rats) for the analyses. Linear regression analyses evaluated correlations between behavior and c-Fos expression, for both good and poor avoiders together, in specific ROIs. All data were $\log _{10}$ transformed prior to statistical analyses due to heterogeneity of variance. $\alpha=$ 0.05 for all analyses.

\section{Acknowledgments}

Research supported by an NIMH grant to J.E.L. (R01 MH38774), an NSF grant to J.E.L. (0920153), a NIDA grant to J.E.L. (R01 DA029053), an NIMH grant to C.K.C. (R21 MH097125), a NIDA subaward to C.K.C. (F6761-01), an NIMH fellowship to C.K.C. (NRSA MH077458), an NIMH fellowship to G.L.-M. (F31MH086294-01A1), an NIMH fellowship to J.M.M. (F32MH094061), and CAPES (\#2350/09-2) and FAPESP grants (\#11/08575-7, 12/06825-9) to R.C.R.M. We thank Hillary Schiff for helpful comments, Claudia Farb and Sneh Kadakia for assistance with histology and immunohistochemistry, and Sanghan Lee and Eva Petkova for aiding with statistical analyses.

\section{References}

Anglada-Figueroa D, Quirk GJ. 2005. Lesions of the basal amygdala block expression of conditioned fear but not extinction. J Neurosci 25: 9680-9685.

Baum M, Poser EG. 1971. Comparison of flooding procedures in animals and man. Behav Res Ther 9: 249-254.

Bolles RC. 1969. Avoidance and escape learning: Simultaneous acquisition of different responses. J Comp Physiol Psychol 68: 355-358.

Bolles RC, Popp RJ Jr. 1964. Parameters affecting the acquisition of Sidman avoidance. I Exp Anal Behav 7: 315-321.

Cain CK, LeDoux JE. 2008. Brain mechanisms of Pavlovian and instrumental aversive conditioning. In Handbook of anxiety and fear, Vol. 17 (ed. Nutt DJ, et al.), pp. 103-125. Elsevier Academic Press, Amsterdam.

Choi JS, Cain CK, LeDoux JE. 2010. The role of amygdala nuclei in the expression of auditory signaled two-way active avoidance in rats. Learn Mem 17: 139-147.

Church RM. 1989. The yoked control design. In Aversion, avoidance and anxiety: Perspectives on aversively motivated behavior (ed. Archer T, Nilsson L), pp. 403-413. Erlbaum, Hillsdale, NJ.

Ciocchi S, Herry C, Grenier F, Wolff SBE, Letzkus JJ, Vlachos I, Ehrlich I, Sprengel R, Deisseroth K, Stadler MB, et al. 2010. Encoding of conditioned fear in central amygdala inhibitory circuits. Nature 468: $277-282$.

Delgado MR, Olsson A, Phelps EA. 2006. Extending animal models of fear conditioning to humans. Biol Psychol 73: 39-48.

Duncan GE, Knapp DJ, Breese GR. 1996. Neuroanatomical characterization of Fos induction in rat behavioral models of anxiety. Brain Res 713: 79-91.

Gozzi A, Jain A, Giovanelli A, Bertollini C, Crestan V, Schwarz AJ, Tsetsenis T, Ragozzino D, Gross CT, Bifone A. 2010. A neural switch for active and passive fear. Neuron 67: 656-666

Han JH, Kushner SA, Yiu AP, Cole CJ, Matynia A, Brown RA, Neve RL, Guzowski JF, Silva AJ, Josselyn SA. 2007. Neuronal competition and selection during memory formation. Science 316: 457-460.

Haubensak W, Kunwar PS, Cai H, Ciocchi S, Wall NR, Ponnusamy R, Biag J, Dong HW, Deisseroth K, Callaway EM, et al. 2010. Genetic dissection of an amygdala microcircuit that gates conditioned fear. Nature 468: $270-276$.

Hefner K, Whittle N, Juhasz J, Norcross M, Karlsson RM, Saksida LM, Bussey TJ, Singewald N, Holmes A. 2008. Impaired fear extinction learning and cortico-amygdala circuit abnormalities in a common genetic mouse strain. I Neurosci 28: 8074-8085.

Herdade KC, Strauss CV, Zangrossi Junior H, Viana MB. 2006. Effects of medial amygdala inactivation on a panic-related behavior. Behav Brain Res 172: 316-323.

Herry C, Mons N. 2004. Resistance to extinction is associated with impaired immediate early gene induction in medial prefrontal cortex and amygdala. Eur J Neurosci 20: 781-790.

Hitchcock JM, Davis M. 1991. Efferent pathway of the amygdala involved in conditioned fear as measured with the fear-potentiated startle paradigm. Behav Neurosci 105: 826-842. 
Kim JJ, Rison RA, Fanselow MS. 1993. Effects of amygdala, hippocampus, and periaqueductal gray lesions on short- and long-term contextual fear. Behav Neurosci 107: 1093-1098.

Knapska E, Radwanska K, Werka T, Kaczmarek L. 2007. Functional internal complexity of amygdala: Focus on gene activity mapping after behavioral training and drugs of abuse. Physiol Rev 87: $1113-1173$.

Lazaro-Munoz G, LeDoux JE, Cain CK. 2010. Sidman instrumental avoidance initially depends on lateral and basal amygdala and is constrained by central amygdala-mediated Pavlovian processes. Biol Psychiatry 67: 1120-1127.

LeDoux JE. 2012. Rethinking the emotional brain. Neuron 73: 653-676.

Levis DJ. 1989. The case for a return to a two-factor theory of avoidance: The failure of non-fear interpretations. In Contemporary learning theories: Pavlovian conditioning and the status of traditional learning theory (ed. Klein SB, Mowrer RR), pp. 227-277. Lawrence Erlbaum Associates, Hillsdale, NJ.

McAllister DE, McAllister WR. 1991. Fear theory and aversively motivated behavior: Some controversial issues. In Fear, avoidance, and phobias: A fundamental analysis (ed. Denny MR). Erlbaum, Hillsdale, NJ.

Moscarello JM, LeDoux JE. 2013. Active avoidance learning requires prefrontal suppression of amygdala-mediated defensive reactions. J Neurosci 33: $3815-3823$.

Mowrer OH, Lamoreaux RR. 1946. Fear as an intervening variable in avoidance conditioning. I Comp Psychol 39: 29-50.

Nikolaev E, Werka T, Kaczmarek L. 1992. C-fos protooncogene expression in rat brain after long-term training of two-way active avoidance reaction. Behav Brain Res 48: 91-94.

Paxinos G, Watson C. 2005. The rat brain in stereotaxic coordinates. Academic Press, Sydney, Australia.

Pitkänen A, Savander V, LeDoux JE. 1997. Organization of intraamygdaloid circuitries in the rat: An emerging framework for understanding functions of the amygdala. Trends Neurosci 20: $517-523$.

Poremba A, Gabriel M. 1999. Amygdala neurons mediate acquisition but not maintenance of instrumental avoidance behavior in rabbits. J Neurosci 19: 9635-9641.
Radwanska K, Nikolaev E, Knapska E, Kaczmarek L. 2002. Differential response of two subdivisions of lateral amygdala to aversive conditioning as revealed by c-Fos and P-ERK mapping. Neuroreport 13: $2241-2246$.

Repa JC, Muller J, Apergis J, Desrochers TM, Zhou Y, LeDoux JE. 2001. Two different lateral amygdala cell populations contribute to the initiation and storage of memory. Nat Neurosci 4: 724-731.

Rescorla RA, Solomon RL. 1967. Two process learning theory: Relationships between Pavlovian conditioning and instrumental learning. Psychol Rev 74: $151-182$.

Saha S, Datta S. 2005. Two-way active avoidance training-specific increases in phosphorylated cAMP response element-binding protein in the dorsal hippocampus, amygdala, and hypothalamus. Eur J Neurosci 21: $3403-3414$

Savonenko A, Filipkowski RK, Werka T, Zielinski K, Kaczmarek L. 1999. Defensive conditioning-related functional heterogeneity among nuclei of the rat amygdala revealed by c-Fos mapping. Neuroscience $\mathbf{9 4 :}$ $723-733$.

Scicli AP, Petrovich GD, Swanson LW, Thompson RF. 2004. Contextual fear conditioning is associated with lateralized expression of the immediate early gene c-fos in the central and basolateral amygdalar nuclei. Behav Neurosci 118: 5-14.

Solomon RL, Wynne LC. 1954. Traumatic avoidance learning: The principles of anxiety conservation and partial irreversibility. Psychol Rev 61: 353.

Sotres-Bayon F, Corcoran KA, Peters J, Sierra-Mercado D. 2008. Neural correlates of individual variability in fear extinction. J Neurosci 28: $12147-12149$.

Yehuda R, LeDoux J. 2007. Response variation following trauma: A translational neuroscience approach to understanding PTSD. Neuron 56: $19-32$.

Yehuda R, Flory JD, Southwick S, Charney DS. 2006. Developing an agenda for translational studies of resilience and vulnerability following trauma exposure. Ann N Y Acad Sci 1071: 379-396.

Received March 8, 2013; accepted in revised form June 4, 2013. 


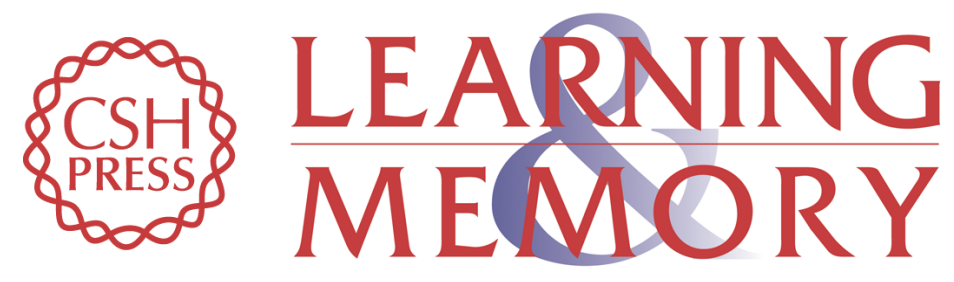

\section{Active vs. reactive threat responding is associated with differential c-Fos expression in specific regions of amygdala and prefrontal cortex}

Raquel C.R. Martinez, Nikita Gupta, Gabriel Lázaro-Muñoz, et al.

Learn. Mem. 2013, 20:

Access the most recent version at doi:10.1101/lm.031047.113

References This article cites 35 articles, 7 of which can be accessed free at: http://learnmem.cshlp.org/content/20/8/446.full.html\#ref-list-1

Creative This article is distributed exclusively by Cold Spring Harbor Laboratory Press for the Commons first 12 months after the full-issue publication date (see

License http://learnmem.cshlp.org/site/misc/terms.xhtml). After 12 months, it is available under a Creative Commons License (Attribution-NonCommercial 3.0 Unported), as described at http://creativecommons.org/licenses/by-nc/3.0/.

Email Alerting Receive free email alerts when new articles cite this article - sign up in the box at the Service top right corner of the article or click here. 